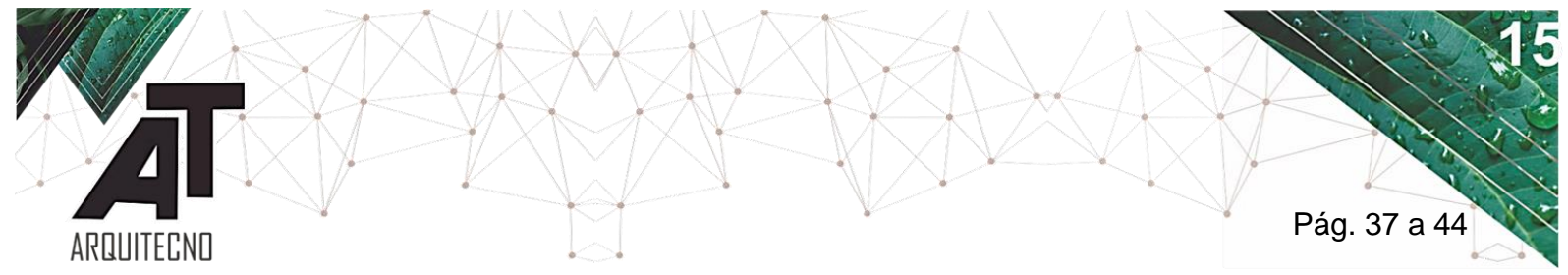

\title{
Anteproyecto de viviendas sociales con Steel Framing en Corrientes. Comparación con sistema húmedo tradicional
}

\section{Social housing steel framing preliminary plan located in Corrientes. Comparison with traditional masonry system}

\author{
Victoria Arengo Piragine, Juan Cruz Breard, Claudia Pilar \\ Facultad de Ingeniería, Universidad Nacional del Nordeste, Argentina \\ vickyarengo@gmail.com; juancruzbreard@gmail.com; claudiapilar2014@gmail.com
}

\section{RESUMEN}

El presente trabajo tiene por objetivo la comparación del sistema húmedo tradicional y el sistema de construcción en seco steel framing, de un conjunto de viviendas de interés social.

El abordaje metodológico consistió en la elaboración del anteproyecto para la ciudad de Corrientes, (diseño arquitectónico, estructural, instalaciones convencionales y no convencionales, verificación de condiciones de habitabilidad) y la comparación entre ambos sistemas teniendo en cuenta las siguientes variables: costo, rapidez de ejecución, mano de obra, confort higrotérmico, ahorro energético, superficie útil, instalaciones, mantenimiento y evaluación social.

Los principales resultados muestran un mejor comportamiento comparativo del sistema en seco por sobre el húmedo, en numerosas variables de análisis. Por lo que su aplicación resulta viable para la construcción de barrios de viviendas sociales para las condiciones de análisis.

\section{ABSTRACT}

The following project's purpose is the comparison of the traditional masonry building system and dry construction with steel framing, in a low-income housing.

The methodology consisted in the elaboration of the preliminary plan located in the city of Corrientes taking in consideration architectural and structural design, installations and the comparison between both systems in the following variables: costs, construction time, labour, hygrothermic confort, energetic savings, maintenance and social response.

The study shows mainly better results for the dry system over the traditional one in multiple variables of the ones analyzed. Taking this as a basis, it is shown that steel framing is a viable system for the construction of a neighbourhood of low-income housing, in the conditions proposed.

PALABRAS CLAVES: sistema industrializado - construcción en seco- sustentabilidad ambiental.

KEY WORDS: steel framing - dry construction - low-income housing

FECHA DE RECEPCIÓN: 26/05/2020 | FECHA DE ACEPTACIÓN: 02/06/2020

DOI: http://dx.doi.org/10.30972/arq.0154386 
Anteproyecto de viviendas sociales con Steel Framing en Corrientes. Comparación con sistema húmedo tradicional

Arengo Piragine, Breard, Pilar

\section{INTRODUCCIÓN}

El presente trabajo tiene por objetivo la comparación entre un conjunto de viviendas de interés social construido con el tradicional sistema húmedo y un sistema de construcción en seco con perfiles metálicos, conocido como steel framing.

El programa arquitectónico abordado (conjunto de viviendas) se fundamenta en el continuo incremento poblacional de la ciudad de Corrientes Capital que, según los censos realizados en los años 2001 y 2010, fue de $8,93 \%$.

Los sistemas constructivos elegidos para la comparación son: el sistema húmedo a base de mampuestos y el steel framing, que significa "bastidor o estructura de acero".

La construcción húmeda tradicional es un sistema constructivo formado por una estructura de paredes portantes, constituidas por mampuestos de diferentes materiales relacionados entre sí mediante morteros y elementos rigidizantes de hormigón armado. Utiliza comúnmente materiales como el hormigón como soporte estructural o bien, paredes portantes de ladrillos, bloques o piedras, materiales que también cumplen el rol de cerramientos.

La obra húmeda es el sistema de construcción más utilizado en Argentina y el más antiguo y, hasta el día de hoy, los planes de vivienda sociales plantean este sistema como método de construcción a pesar de su baja aptitud frente al confort higrotérmico.

En el lado opuesto de la comparación se encuentra el steel framing, sistema constructivo que consiste en una estructura de perfiles de chapa de acero de bajo espesor separados cada 40 o 60 centímetros, que, junto a distintos paneles y elementos, definen el cerramiento, funcionando todo como un conjunto resistente. Los elementos que forman el esqueleto estructural se vinculan para soportar las cargas del edificio.

El trabajo de investigación se justifica por el nuevo escenario que surge por la promulgación de la Resolución 5-E-2018 de la Secretaría de Vivienda y Hábitat, a principios de 2018, por la cual el Steel framing pasa a ser construcción tradicional y no requiere Certificado de Aptitud Técnica (CAT) siempre y cuando el cálculo estructural siga lo establecido en el Reglamento CIRSOC 303 y la norma IRAM IAS U 500-205. Además, se tienen en cuenta la actualización de los "Estándares mínimos de calidad para viviendas de interés social", del año 2017, que establece mayores exigencias, que en general no son alcanzadas en los métodos tradicionales, por lo que el steel framing, aparece como una opción atractiva que resulta necesario verificar mediante parámetros objetivos.

\section{DESARROLLO}

El anteproyecto se encuentra emplazado en el barrio Ex - Aeroclub de la ciudad de Corrientes, ocupando un terreno de 14 hectáreas. En función del tipo de distrito en el que se encuentra el mismo y de las normativas locales vigentes se toma un lote tipo de 12,5 metros de frente por 25 metros de fondo. Como usuario se define una familia tipo de cuatro integrantes. El programa arquitectónico consta de estar comedor, cocina, dos dormitorios, baño, lavadero y estacionamiento semicubierto. El dimensionamiento de los locales tiene en cuenta la modulación que deben seguir los perfiles metálicos en el sistema constructivo steel framing, eligiendo separaciones de 60 centímetros, y la intención de reducir desperdicios haciendo un uso óptimo de las placas de cerramientos exteriores, evitando los cortes innecesarios.

Considerando lo dicho anteriormente y, respetando las características mínimas a cumplir según el Código de Edificación de la ciudad de Corrientes y la Norma de Estándares Mínimos de Calidad para Viviendas de Interés Social, se define una vivienda de 74,34 m2 considerando los espacios semicubiertos. En la figura 1 se observa una imagen de la fachada propuesta para las viviendas, en la figura 2 a la izquierda la planta arquitectónica y a la derecha la propuesta de materialización tecnológica. 


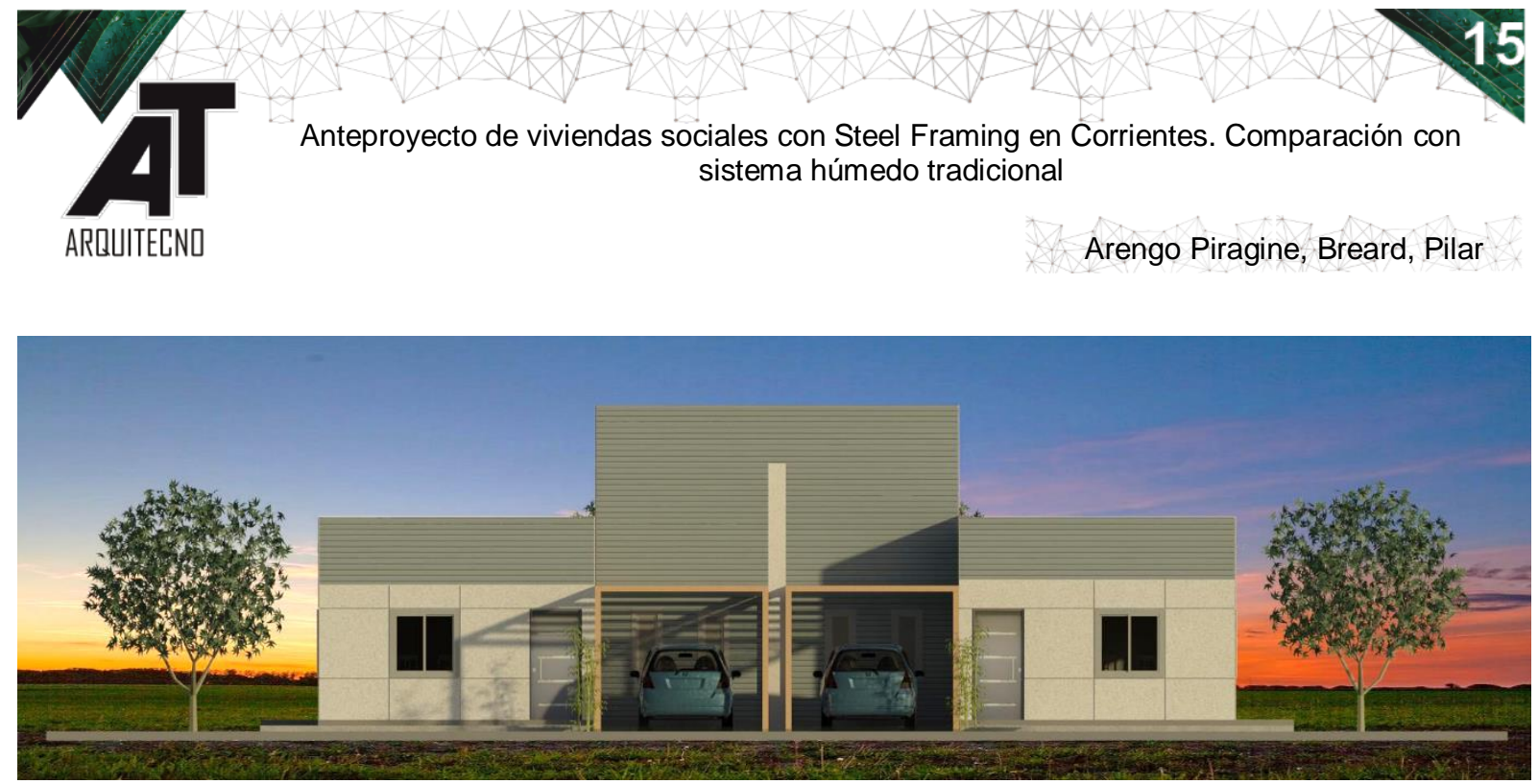

Figura 1: Fachada de las viviendas apareadas propuestas para su análisis. Fuente: Arengo y Breard, 2018.
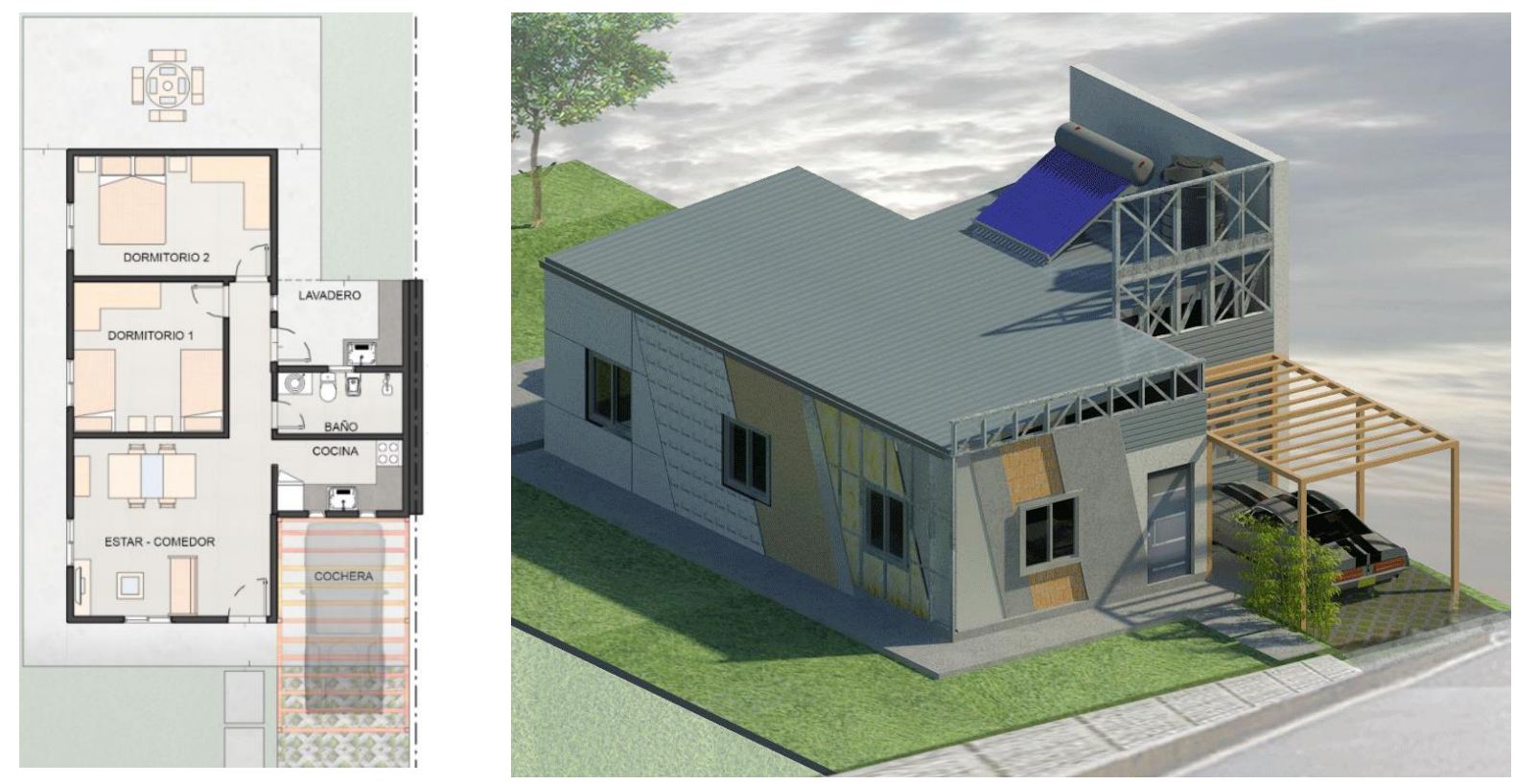

Figura 2: A la izquierda planta arquitectónica base y a la derecha axonométrica de la resolución tecnológica adoptada. Fuente: Arengo y Breard, 2018.

De acuerdo a la norma IRAM 11.603 la ciudad de Corrientes se encuentra ubicada en la zona lb (muy cálida húmeda). Por ello en el diseño se priorizaron la orientación este - noreste - norte, y en los casos que la tipología quedara mal orientada se aplicaron mecanismos de control solar a través de galerías, parasoles verticales y el uso selectivo de la vegetación de hoja caduca.

Para restringir el intercambio energético entre interior y exterior se opta por una disposición de semi perímetro libre para limitar la transferencia de energía, pero posibilitando al mismo tiempo la ventilación cruzada, que resulta benéfica para el clima muy cálido húmedo. Esta configuración de viviendas "apareadas" permitió levantar la pared medianera entre ellas, materializada en mampostería tradicional, sobre la cual apoyará el tanque de reserva. Éste muro medianero, brinda a su vez, rigidez al prototipo realizado con steel framing.

\section{Análisis estructural}

Para posibilitar el análisis comparativo de ambas viviendas el tipo de fundación adoptada es una platea de hormigón (compatible con ambos sistemas, con adecuada seguridad estructural y con un costo razonable) con vigas perimetrales para su rigidización en coincidencia con las principales paredes portantes. También se consideran dos espesores de la losa de fundación, de menores dimensiones a 


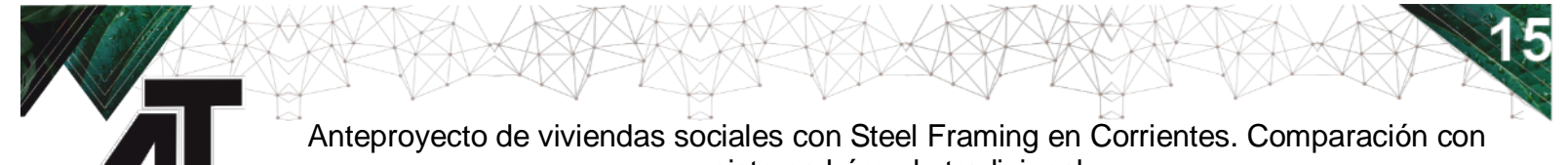

Anteproyecto de viviendas sociales con Steel Framing en Corrientes. Comparación con sistema húmedo tradicional

ARQUITECNO

Arengo Piragine, Breard, Pilar

los alrededores de la vivienda, generando una vereda perimetral, que resulta una medida preventiva para evitar la humedad de cimientos.

Una vez dimensionada la fundación se procede al análisis estructural de la vivienda. Al ser un prototipo de una sola planta, no se realiza un cálculo para el prototipo tradicional, solo se reprodujo la misma teniendo como base una vivienda propuesta por el Instituto de Vivienda de Corrientes (INVICO). Por otro lado, se realiza el análisis estructural de los perfiles que componen el entramado metálico del prototipo con steel framing. Al consistir éste en una estructura muy liviana se tienen en mayor consideración las cargas de succión generadas por la acción del viento que las gravitatorias por su peso propio.

Para poder dar una mayor resistencia a los efectos del viento, se realiza el análisis estructural considerando un conjunto de dos casas con pendientes de un agua hacia los lados unidas en el medio por un muro medianero de ladrillos comunes. Queda generada así una estructura con pendiente a dos aguas con un elemento rigidizador en el medio de éstas, que se analiza ante la acción del viento en dos direcciones principales según el Reglamento argentino de vientos CIRSOC 102.

En el cálculo y verificación de los perfiles se toma como hipótesis que sólo la estructura metálica es la encargada de resistir todos los esfuerzos a los que está sometida la vivienda. Se estudian uniones entre los elementos metálicos y los paneles a reforzar con sus respectivas cargas en función de la combinación de éstas que generan los efectos más perjudiciales en la estructura, en búsqueda de los perfiles que se encuentran más solicitados a cada uno de los respectivos esfuerzos. Una vez identificados se calcula sus resistencias de diseño en función de lo establecido en el reglamento CIRSOC 303, buscando que sean mayores a las resistencias requeridas.

\section{Verificación higrotérmica}

En primera instancia se definen los cerramientos verticales por tipología: muro tradicional de ladrillos cerámicos huecos de $18 \mathrm{~cm}$ de espesor revocados a ambas caras y el steel framing con las siguientes capas: placas de yeso e $=12,5 \mathrm{~mm}$, aislación térmica de lana de vidrio con foil de aluminio de $50 \mathrm{~mm}$, placas OSB de $9 \mathrm{~mm}$, barrera de agua y viento y placa cementicia de $10 \mathrm{~mm}$.

Se realiza la verificación higrotérmicas de los cerramientos exteriores, teniendo en cuenta la transmitancia térmica $(\mathrm{K})$ y el riesgo de condensaciones (superficiales e intersticiales) de acuerdo a los parámetros establecidos en los "Estándares Mínimos de Calidad para Viviendas de interés social", en ambas tipologías (tradicional y Steel Framing).
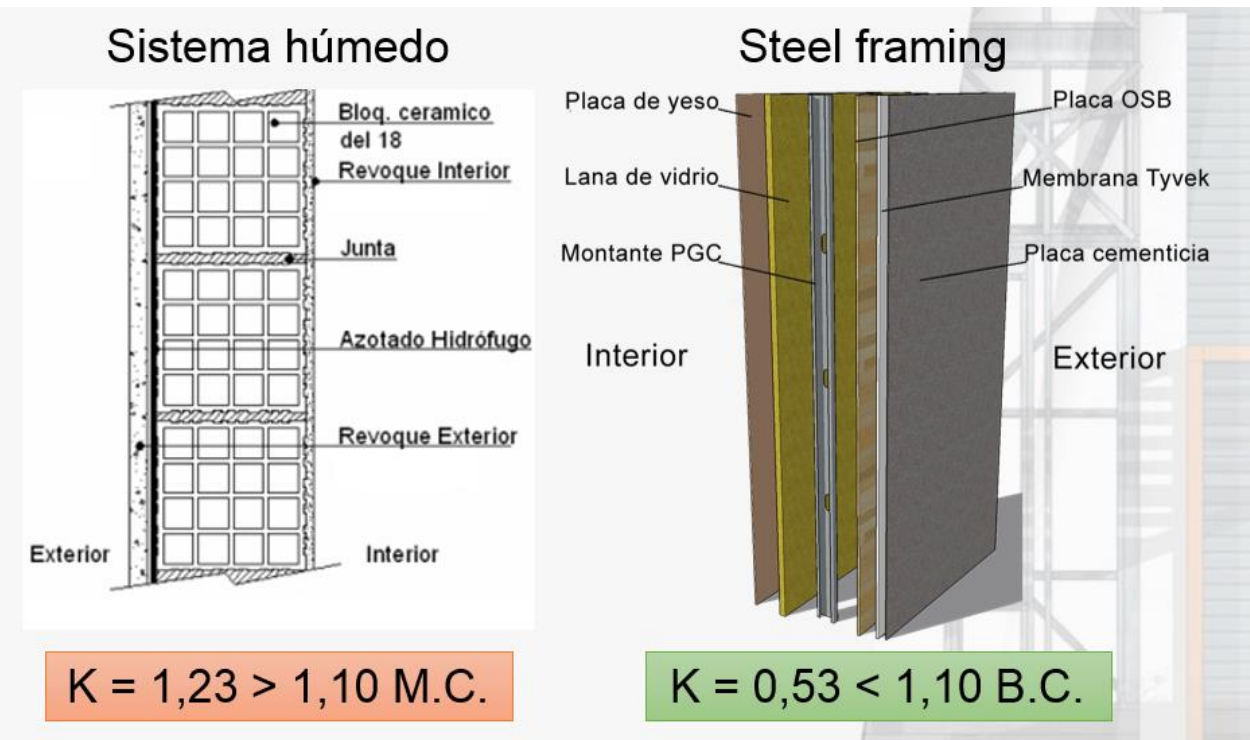

Figura 3: Resultados de cálculo de K a la izquierda del muro tradicional y a la derecha el cerramiento vertical de Steel Framing. Fuente: Arengo y Breard, 2018. 
Anteproyecto de viviendas sociales con Steel Framing en Corrientes. Comparación con sistema húmedo tradicional

En la figura 3 se observa que el muro tradicional da por resultado un $\mathrm{K}$ de diseño de 1,23 W/m2k (no verifica al nivel mínimo $C$ que establece un $\mathrm{K}$ admisible de $1,10 \mathrm{~W} / \mathrm{m} 2 \mathrm{k}$ ) mientras que el steel framing posee un $\mathrm{K}$ de diseño de $0,53 \mathrm{~W} / \mathrm{m} 2 \mathrm{k}$, verificando a nivel medio $\mathrm{B}$ (que define un $\mathrm{K}$ admisible de 0,53 $\mathrm{W} / \mathrm{m} 2 \mathrm{k}$ ). Por otro lado, se calcula el riesgo de condensación en función de las temperaturas interiores y exteriores de la vivienda según lo establece la norma IRAM 11.625, también para ambos sistemas, verificando nuevamente solo la de steel framing.

En cuanto al diseño de la cubierta, se definen iguales aislaciones para ambos casos: una aislación térmica con barrera de vapor inmediata al cielorraso (lana de vidrio con foil de aluminio e $=50 \mathrm{~mm}$ ) y, debajo de la chapa de la cubierta, una membrana termohidrófuga (membrana tipo ISOLANT e $=10 \mathrm{~mm}$ ). Además, como estructura de la propuesta húmeda se dispone de correas metálicas, mientras que en el de steel framing se plantean cabreadas compuestas por perfiles galvanizados de pequeño espesor, al igual que el resto de los perfiles del sistema.

Con todos los elementos de la cubierta determinados, se calcula el coeficiente de transmitancia térmica resultando $\mathrm{K}=0,57$, menor que la admisible para el confort higrotérmico de nivel $\mathrm{B}$, logrando cumplir con condiciones de confort media, exigido por los "Estándares mínimos de calidad para viviendas de interés social".

Sin embargo, a la hora de analizar el sistema de perfilería metálica, hay que tener en cuenta los puentes térmicos, siendo estos los puntos de la envolvente donde se interrumpe la continuidad de la aislación térmica generando transmitancia térmica no deseada como lo son los montantes de la estructura. La norma IRAM 11.605 limita la relación entre el coeficiente $\mathrm{K}$ del puente térmico y el coeficiente $\mathrm{K}$ de un muro opaco siendo la admisible de 1,35 mientras que en el prototipo analizado la misma resultó ser de 2,26 , no verificando. Una solución para este inconveniente sería la utilización de una segunda aislación térmica lo que incrementa los costos.

Si bien los nombrados coeficientes ya caracterizan térmicamente a las viviendas en sus dos versiones por medio de sus cerramientos, se decide proseguir con un análisis global que considere también las transferencias de calor por radiación solar, fuentes internas y ventilación, además de tener en cuenta las aberturas existentes.

Siguiendo el procedimiento establecido en la norma IRAM 11.659, se calcula la carga térmica total de refrigeración de cada tipología de vivienda en análisis, dando como resultado que la vivienda tradicional no verifica mientras que la vivienda con steel framing se encuentra dentro de los límites admisibles. Estos valores también permiten determinar la cantidad y potencia de equipos de acondicionamiento necesarios para cada vivienda. En dicho análisis resulta que para la vivienda realizada con perfilería metálica se necesitan equipos de menor potencia.

\section{Aspectos constructivos}

Para analizar la rapidez de ejecución se realiza un estudio de los distintos rubros requeridos para materializar la vivienda en ambos sistemas constructivos y cuánto tiempo demanda cada uno de ellos, para así poder determinar el tiempo total de terminación de la obra.

Se toma para el análisis un conjunto de 10 viviendas. El plazo de ejecución de la vivienda tradicional es de 6 meses, mientras que la de Steel framing se estima en 4 meses, lo que significa una disminución del plazo de $33 \%$.

En cuanto a las instalaciones (eléctricas y sanitarias) la construcción tradicional resulta poco eficiente dado que se realizan canaletas para embutir las cañerías lo que insume mayor gasto de material, mayor insumo de de mano de obra y genera mayor volumen de residuos de obra que solo en limitadas ocasiones es reutilizado, generando gastos adicionales para su gestión y disposición final. Por su parte el steel framing tiene prevista la disposición de instalaciones entre perfiles. Desde un punto de vista positivo se disminuye notablemente la generación de residuos y acelera los plazos de ejecución; desde un punto de vista negativo, la mano de obra posiblemente requiera de un mayor grado de especialización.

Otro aspecto analizado ha sido la necesidad de mantenimiento. La tipología tradicional requiere de pintura periódica, con mano de obra no especializada. Por su parte, el steel framing podría exigir un 
Anteproyecto de viviendas sociales con Steel Framing en Corrientes. Comparación con sistema húmedo tradicional

tomado de juntas de las placas de yeso interior y la renovación de pintura cada 5 años de paramentos interiores y exteriores. Un aspecto a considerar es la mayor "fragilidad" de los cerramientos de Steel Framing, frente a un uso inadecuado por golpes fuertes de muebles o vehículos.

\section{Costos}

El primer paso ha sido el cómputo métrico de cada uno de los sistemas, subdividiendo los trabajos de la obra en diferentes rubros, y éstos a su vez, en distintos ítems elementales agrupados y ordenados según la secuencia lógica de ejecución.

Ejecutado el cómputo de cada vivienda, se procede a realizar el costo - costo mediante el método de análisis de precios unitarios, discriminando el costo de los materiales y el de la mano de obra.

Para el análisis del costo de los materiales se realizan consultas a proveedores locales y revistas especializadas (Arquitectura \& Construcción №398, noviembre 2018), mientras que para el análisis del costo de la mano de obra se obtiene multiplicando la cantidad de horas necesarias para la ejecución de la obra por ítem por el jornal real del obrero.

En el caso de steel framing, se consideran obreros con el rango de oficiales especializados, mientras que las horas necesarias de ejecución de cada tarea se calculan en base al rendimiento provisto por empresas privadas que se dedican a realizar el sistema en la región.

El costo - costo se calcula entonces sumando los costos de material más los costos por mano de obra por rubro. El costo es el resultado de la suma del costo - costo y los gastos generales de la obra. En estos gastos generales se incluyeron por un lado los gastos generales directos, propios de la obra y los gastos generales indirectos, propios de la empresa.

Como el trabajo planteado es a nivel de anteproyecto, se toma la simplificación de que los gastos generales representan aproximadamente un 15\% del costo - costo. Sin embargo, esta simplificación aplicada a ambos sistemas no permite ver la reducción en gastos generales directos mensuales que se obtiene por la reducción de tiempos de obra para el caso del prototipo realizado con perfilería metálica. Para tener en cuenta entonces la rapidez de ejecución, consideramos realizar la simplificación de adoptar como gastos generales un $10 \%$ del costo - costo en el cálculo de costo del prototipo con steel framing. Finalmente, se calcula el precio de oferta donde están incluidos todos los costos de la obra en sí y del beneficio que recibe la empresa, los impuestos que se deben abonar como ser el Impuesto al valor agregado (IVA) e ingresos brutos. En la tabla 1 puede observarse los distintos valores.

\begin{tabular}{lcccccccc}
\hline $\begin{array}{c}\text { Sistema } \\
\text { constructivo }\end{array}$ & $\begin{array}{c}\text { Costo } \\
\text { Costo }\end{array}$ & GG & Costos & $\begin{array}{c}\text { Bene- } \\
\text { ficios }\end{array}$ & & Precio & $\begin{array}{c}\text { Impues } \\
\text { tos }\end{array}$ & \multicolumn{2}{c}{ Precio de oferta } \\
\hline Tradicional & 939.370 & 140.905 & 1.080 .276 & 108.027 & 1.188 .303 & 294.105 & 1.482 .408 & 22.687 \\
\hline Steel Framing & 1.053 .535 & 105.353 & 1.158 .888 & 115.888 & 1.274 .777 & 315.507 & 1.590 .284 & 24.338 \\
\hline
\end{tabular}

Tabla 1: Síntesis del análisis de costo de ambos sistemas. Fuente: Arengo y Breard, 2018.

Luego del análisis de los precios, se realiza la evaluación del anteproyecto para ver su conveniencia de implementación a nivel social. Para las evaluaciones, se consideran como beneficios sociales los valores de las viviendas antes de ser aplicados los impuestos. Por otro lado, a la evaluación del anteproyecto del barrio de viviendas con steel framing se le agrega el beneficio que se obtuvo por ahorro energético en equipos de acondicionamiento con respecto al del sistema húmedo tradicional. Para un flujo de beneficios netos para todo el conjunto de viviendas, con un horizonte de 20 años, considerando $12 \%$ como tasa de oportunidad, se calculan finalmente los Valores Actuales Netos (VAN) para ambos sistemas, cuyos valores se transcriben en la tabla 2.

\begin{tabular}{cc}
\hline \multicolumn{2}{c}{ VAN $_{12 \%}$ social } \\
\hline Construcción tradicional & Steel framing \\
\hline$\$ 63.538 .647$ & $\$ 98.515 .120$ \\
\hline
\end{tabular}

Tabla 2: Valores del VAN para una tasa de oportunidad del 12\%. Fuente: Arengo y Breard, 2018. 


\section{Comparación de sistemas}

En cuanto a los costos el steel framing resulta más caro en un $7 \%$, lo que se puede ver contrarrestado por un aumento de la rapidez de ejecución que ronda el 33\% y la superficie útil que aumenta en un 3\%. Desde el punto de vista de la eficiencia energética, el cerramiento vertical tradicional no verifica a ningún nivel de confort, por lo que no cumple con los "Estándares mínimos de calidad para viviendas de interés social". Por su parte el steel framing verifica a nivel de confort b (medio), cumpliendo con los parámetros establecidos. Esto trae como consecuencia una menor carga de refrigeración (calculada a través de la norma IRAM 11.659). Las instalaciones pueden considerarse tanto un aspecto a favor para el steel framing (menor generación de residuo y menor plazo de ejecución) y negativo (posible necesidad de capacitación de la mano de obra).

En la tabla 3 se realiza una síntesis comparativa del sistema tradicional y el Steel framing en la última columna un signo positivo (+) señalando los aspectos en los que el Steel framing posee un mejor desempeño y con signo negativo (-) los aspectos que resultan negativos.

\begin{tabular}{|l|c|c|l|c|}
\hline \multicolumn{1}{|c|}{ Aspecto } & $\begin{array}{c}\text { Construcción } \\
\text { Tradicional }\end{array}$ & $\begin{array}{c}\text { Steel } \\
\text { Framing }\end{array}$ & \multicolumn{2}{c|}{$\begin{array}{l}\text { Valoración del Steel framing en } \\
\text { relación a la construcción tradicional }\end{array}$} \\
\hline $\begin{array}{l}\text { Costo/m2 } \\
\text { (a diciembre de 2018) }\end{array}$ & $22.688 \$ / \mathrm{m} 2$ & $24.339 \$ / \mathrm{m} 2$ & $\begin{array}{l}\text { Mayor costo. Incremento de 1.651 } \\
\$ / \mathrm{m} 2(+7 \% \text { más caro) }\end{array}$ & - \\
\hline Plazo de ejecución & $6 \mathrm{meses}$ & $4 \mathrm{meses}$ & Menor plazo $(-33 \%)$ & + \\
\hline Superficie útil & $58,65 \mathrm{~m} 2$ & $60,48 \mathrm{~m} 2$ & Mayor superficie $(+3,02 \%)$ & + \\
\hline $\begin{array}{l}\text { K de verano } \\
\text { Cerramientos verticales }\end{array}$ & $1,2 \mathrm{~W} / \mathrm{mk}$ & $0,53 \mathrm{~W} / \mathrm{mk}$ & Menor K & + \\
\hline $\begin{array}{l}\text { Confort Higrotérmico } \\
\text { cerramientos verticales }\end{array}$ & $\begin{array}{c}\text { No verifica } \\
\text { a ningún nivel }\end{array}$ & $\begin{array}{c}\text { Verifica } \\
\text { a Nivel B }\end{array}$ & Mayor nivel de confort & + \\
\hline Carga de refrigeración & $5.922 \mathrm{~W}$ & $4.777 \mathrm{~W}$ & $\begin{array}{l}\text { Menor consumo energético } \\
(-23 \%)\end{array}$ & + \\
\hline \multirow{2}{*}{ Instalaciones } & Amuradas & Entre perfiles & Menos residuos de obra & + \\
\cline { 5 - 6 } & & & Mano de obra más especializada & - \\
\hline
\end{tabular}

Tabla 3: comparación entre el sistema tradicional y el Steel framing. Fuente: elaboración propia.

\section{CONCLUSIONES}

A través del análisis de múltiples aspectos se puede verificar la factibilidad de implementación del steel framing para la construcción de un conjunto de viviendas sociales ya que, para las distintas prestaciones que posee, la diferencia en el costo por metro cuadrado de vivienda con respecto al sistema húmedo tradicional es solo de un $6,8 \%$. Por otro lado, los valores de VAN positivos indican la conveniencia de ambos proyectos desde el punto de vista social, mientras que la diferencia en el valor entre ambos sistemas se remite a la eficiencia energética que posee la vivienda con perfilería metálica. El sistema constructivo steel framing destaca su conveniencia en los aspectos de rapidez de ejecución, confort higrotérmico, ahorro energético y mayor superficie útil por reducción de espesores de muros. Su principal atributo resulta ser su rapidez de ejecución, que, para el prototipo analizado, es de 33\% menor a la de la construcción tradicional. Este distintivo permitiría su aplicabilidad para situaciones de emergencia, en donde el tiempo representa un factor crítico.

En el lado opuesto, el sistema húmedo tradicional se aventaja con respecto al otro sistema por costos y mano de obra existente en el mercado local, sin necesidad de invertir en capacitación de trabajadores. Adicionalmente, steel framing es una metodología que requiere de una mayor cantidad de planos de detalles por la complejidad de las uniones de los elementos constituyentes del sistema.

Finalmente, como la incidencia de los materiales en el costo total de la vivienda representa un valor muy alto, por lo que la competitividad del sistema steel framing está sujeta a la variabilidad de los precios de los mismos. 
